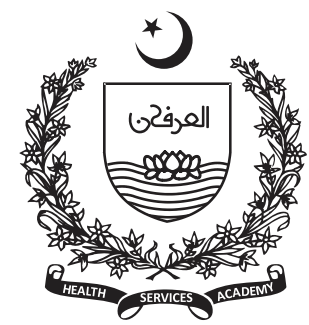

Division of Clinical and Translational Research, Larkin Community Hospital, South Miami, FL, USA

Internal Medicine, Jinnah Medical and Dental College, Karachi, Pakistan

Corresponding Author: Uzzam Ahmed Khawaja Email: uzzamahmedkhawaja@gm ail.com

\section{Psychological Impact of the COVID-19 Pandemic on the Mental Health Status of Health Care Workers (HCW) in Pakistan}

\author{
Uzzam Ahmed Khawaja
}

$\mathrm{T}$

he COVID-19 outbreak has affected physical health along with mental health, but sadly, physical health till now has been the center of attention (1). Amid the pandemic, health care workers $\left(\mathrm{HCW}^{\prime} \mathrm{s}\right)$ are at greater risk of stress and anxiety due to increased working schedules without adequate periods of rest, the fright of getting infected because of close contact with the infected patients, and disrupted nap routines (1). In February 2020, the COVID-19 pandemic began to spread across Pakistan (2). This not only took the entire country by storm but also put the competency of the health care system to test.

Sandesh et al. conducted a study across various hospitals in Karachi, Pakistan to evaluate the mental health status of HCW's deployed in COVID-19 isolation wards and according to their findings, 81 $(72.3 \%)$ participants underwent moderate to extreme levels of severe depression, 96 (85.7\%) participants encountered moderate to extremely severe anxiety, and 101 (90.1\%) participants faced moderate to extreme levels of stress (3). Additional noteworthy findings of the study included stress and anxiety among HCW's managing COVID-19 infected patients being the most common cause of fear of infecting members of their family $(89.2 \%)$, the terror of getting themselves infected (80.3\%). Other contributing factors included greater workload $(64.2 \%)$, a dearth of PPE (62.5\%), a lack of security $(62.5 \%)$, and a lack of public awareness regarding COVID-19 (46.4\%) (3). Other factors that contribute to an increasing prevalence of anxiety, depression, and stress among HWC's in Pakistan are a frail infrastructure for patient care, lack of public awareness, and penurious compliance with safety measures (3). The compromised mental health status of an HCW can have alarming consequences that can have long term implications such as altered immunity, thus increasing the vulnerability to diseases (4). This, therefore, prompts an urgent need to establish measures to safeguard the mental well-being of HCW (5). Through this letter we emphasize on evaluating the mental health status and well-being of HCW's striving to survive the COVID-19 pandemic wave, caring for their COVID-19 positive patients, and practicing measures to help ease their mental health burden.

Financial support and sponsorship: Nil.

Conflicts of interest: None. 
Psychological Impact of the COVID-19 Pandemic on the Mental Health Status of Health Care Workers (HCW) in Pakistan

\section{References}

1. S. Khan, G. Nabi, G. Han et al. Novel coronavirus: how the things are in Wuhan Clin Microbiol Infect (2020), 10.1016/j.cmi.2020.02.005

2. Khalid, A., Ali, S. COVID-19 and its Challenges for the Healthcare System in Pakistan. ABR (2020). https://doi.org/10.1007/s41649-020-00139-x

3. Sandesh R, Shahid W, Dev K, et al. Impact of COVID-19 on the Mental Health of Healthcare Professionals in Pakistan. Cureus. 2020;12(7):e8974. Published 2020 Jul 2. doi:10.7759/cureus.8974

4. J.J. Chiang, S.W. Cole, J.E. Bower et al. Brain, Behavior, and Immunity Depressive symptoms and immune transcriptional profiles in late adolescents Brain Behav Immun, 80 (2019), pp. 163-169, 10.1016/j.bbi.2019.03.004

5. Moazzami, B.; Razavi-Khorasani, N.; Moghadam, A.D.; Farokhi, E.; Rezaeia, N. COVID-19 and telemedicine: Immediate action required for maintaining healthcare providers well-being. J. Clin. Virol. 2020, 126, 104345 